\title{
Sometimes less is more in multiple sclerosis drug switching
}

\author{
Às vezes menos é mais na troca de medicações em esclerose múltipla \\ Bruna Klein da Costa ${ }^{1}$, Douglas Kazutoshi Sato ${ }^{1,2,3}$
}

\author{
1Pontifícia Universidade Católica \\ do Rio Grande do Sul, Hospital \\ São Lucas e Instituto do Cérebro, \\ Serviço de Neurologia, Porto Alegre \\ RS, Brasil; \\ ¿Universidade de São Paulo, \\ Faculdade de Medicina, \\ Departamento de Neurologia, São \\ Paulo SP, Brasil; \\ ${ }^{3}$ Tohoku University Graduate \\ School of Medicine, Department of \\ Neurology, Sendai, Japan.
}

\section{Correspondence:}

Douglas Kazutoshi Sato; Pontificia

Universidade Católica do Rio

Grande do Sul; Av Ipiranga, 6690;

90610-000 Porto Alegre RS, Brasil;

E-mail:douglas.sato@pucrs.br

Conflict of interest:

Dr da Costa has no conflict of interest related to this article.

Dr Sato has received speaker

honoraria from Biogen and Novartis.

Received 13 July 2016;

Accpeted 20 July 2016.
T

he treatment options for relapsing-remitting multiple sclerosis (RRMS), a chronic central nervous system inflammatory demyelinating disorder, have widened in the past decades. Nowadays, medications can significantly reduce the relapse rate and magnetic resonance disease activity even in patients with highly inflammatory disease courses. Among the options for non-responders to first line drugs or aggressive onset RRMS is natalizumab, a recombinant humanized monoclonal antibody against alpha-4 integrin that interferes with immune cell transmigration across the endothelial layer at the blood-brain-barrier ${ }^{1}$. Natalizumab may reduce annual relapse rate in $68 \%$ and disability progression in $42 \%$ compared to placebo ${ }^{2}$.

Despite its effects in disease control, patients under treatment with natalizumab are at risk of developing progressive multifocal leukoencephalopathy (PML) caused by JC virus (JCV) reactivation, especially those with high-index serum antibody positivity, treatment courses above 24 months, and previous treatment with immunosuppressive agents ${ }^{3}$. Furthermore, patients submitted to long-term treatment with natalizumab discontinue the drug due to other factors such as drug intolerance, neutralizing antibodies production and unsatisfactory therapeutic response. An alternative treatment of natalizumab in this scenario is fingolimod, which also acts via immune cell trafficking, albeit via antagonism of the sphinosine-1-phosphate receptor family, resulting in lymphocyte sequestration within lymph nodes ${ }^{4}$. However, a washout period is thought to be necessary when switching from natalizumab to fingolimod given their potentially synergistic mechanisms, which could expose the patients to a higher risk of opportunistic infections. In this period, the patients with highly active disease remain exposed to the risk of exacerbations.

Several studies have been evaluating the adequate washout period that reduces the relapse risk without increasing prohibitively the risks of infection. Initially the recommendation was three to six months, although this time has been shortened progressively until 8 to 12 weeks 5 . In this issue of Arquivos de Neuropsiquiatria, Fragoso et al. ${ }^{6}$ evaluated the safety and disease control of $25 \mathrm{JCV}$ positive patients submitted to an even shorter washout period of 4 to 8 weeks. Favoring earlier discontinuation, Comi et al. ${ }^{7}$ analyzed the recurrence rate of patients in fingolimod treatment that previously received natalizumab and found that the increase in disease activity followed by natalizumab suspension significantly decrease when fingolimod was initiated. Another observational survey-based study from France suggested that the washout period could be shorter than 3 months 8 .

A matter of concern is that after 4 weeks of natalizumab treatment cessation, antibody concentration in serum is still detectable ${ }^{9}$. Therefore, fingolimod treatment initiation after only 4 weeks would result in overlapping exposure to natalizumab and fingolimod. The immunological consequences of drug switching are still not completely understood. Klotz et al. ${ }^{9}$ designed a study protocol that intend to evaluate the changes in immune function over time during the treatment switch (particularly in the expression of CD49d and CD62L and the migratory capacity of peripheral immune cells and anti-viral immune responses that predispose natalizumab and fingolimod to different types of virus reactivation or infection). However, it is important to keep in mind that this could represent a low risk of opportunistic infection in clinical practice, since the study conducted by Fragoso et al does not found major safety issues. 
The great amount of options for treatment of RRMS has brought not only the challenges of choosing the right treatment to the right patient and the safety issues of each isolated drug, but also the hard task of better understanding the interactions between them and the properly time to change treatment and how to do it. Maybe, with the elucidation of the exact immunological effects of switching disease-modifying-treatments, we could better establish the middle term between the risk of recurrence and opportunistic infections. What we know from the acquired experience with these drugs is that shorter washout periods between treatments are likely to be safe and used in selected patients with highly active RRMS.

\section{References}

1. Miller DH, Khan OA, Sheremata WA, Blumhardt LD, Rice GPA, Libonati MA et al. A controlled trial of natalizumab for relapsing multiple sclerosis. N Engl J Med. 2003;348(1):15-23. doi:10.1056/NEJMoa020696

2. Polman $\mathrm{CH}, \mathrm{O}$ 'Connor PW, Havrdova E, Hutchinson M, Kappos $\mathrm{L}$, Miller DH et al. A randomized, placebo-controlled trial of natalizumab for relapsing multiple sclerosis. N Engl J Med. 2006;354(9):899-910. doi:10.1056/NEJMoa044397

3. Bloomgren G, Richman S, Hotermans C, Subramany M, Goetz S, Natarajan A et al. Risk of natalizumab-associated progressive multifocal leukoencephalopathy. N Engl J Med. 2012;366(20):1870-80. doi:10.1056/NEJMoa1107829

4. Gräler MH, Goetzl EJ. The immunosuppressant FTY720 down-regulates sphingosine 1-phosphate G-protein-coupled receptors. FASEB J. 2004;18(3):551-3. doi:10.1096/fj.03-0910fje

5. Kappos L, Radue EW, Comi G, Montalban X, Butzkueven H, Wiendl $\mathrm{H}$ et al. Switching from natalizumab to fingolimod: A randomized, placebo-controlled study in RRMS. Neurology. 2015;85(1):29-39. doi:10.1212/WNL.0000000000001706.
6. Fragoso YD, Alves-Leon SV, Becker J, briijs JBB, Correa EC, Damasceno A et al. Safety of switching from natalizumab straight into fingolimod in a group of JCV-positive patients with multiple sclerosis. Arq Neuropsiquiatr. 2016;74(8):650-2. doi: 10.1590/0004-282X20160090

7. Comi G, Gold R, Dahlke F, Sinha A, Rosenstiel P, Tomic D, Kappos L et al. Relapses in patients treated with fingolimod after previous exposure to natalizumab. Mult Scler. 2015;21(6):786-90. doi:10.1177/1352458514549404

8. Cohen M, Maillart E, Tourbah A, De Sèze J, Vukusic S, Brassat D et al. Switching from natalizumab to fingolimod in multiple sclerosis: a French prospective study. JAMA Neurol. 2014;71(4):436-41. doi:10.1001/jamaneurol.2013.6240

9. Klotz L, Grützke B, Eveslage M, Deppe M, Gross CC, Kirstein L et al. Assessment of immune functions and MRI disease activity in relapsing-remitting multiple sclerosis patients switching from natalizumab to fingolimod (ToFingo-Successor). BMC Neurol. 2015;15:96. doi:10.1186/s12883-015-0354-9 